\title{
Pain and Kidney Function Decline and Mortality: A Cohort Study of US Veterans
}

Vanessa Ravel, $\mathrm{MPH}^{1}$, Seyed-Foad Ahmadi, MD, MPH ${ }^{1}$; Elani Streja, $\mathrm{MPH}, \mathrm{PhD}^{1}$;

Jonathan A. Sosnov, MD ${ }^{2}$; Csaba P. Kovesdy, MD ${ }^{3}$; Kamyar Kalantar-Zadeh, MD, MPH, $\mathrm{PhD}^{1,4}$; and Joline L.T. Chen, MD, $\mathrm{MS}^{1,4}$.

${ }^{1}$ Harold Simmons Center for Kidney Disease Research and Epidemiology, Division of Nephrology and Hypertension, University of California Irvine Health, Orange, CA

${ }^{2}$ Division of Nephrology, Department of the Air Force $59^{\text {th }}$ Medical Wing (AETC), Joint Base San Antonio-Lackland, Texas

${ }^{3}$ Division of Nephrology, Memphis Veterans Affairs Medical Center, Memphis, TN;

${ }^{4}$ Department of Medicine, Long Beach Veteran Affairs Health System, Long Beach, CA

\section{Correspondence:}

Kamyar Kalantar-Zadeh, MD, MPH, PhD

Harold Simmons Center for Kidney Disease Research and Epidemiology

Division of Nephrology and Hypertension

University of California Irvine, School of Medicine

101 The City Drive South, City Tower, Orange, California 92868-3217

Tel: (714) 456-5142, Fax: (714) 456-6034

Email:kkz@uci.edu

or

Joline LT Chen, MD, MS

Department of Medicine, Long Beach Veteran Affairs Health System, 5801 E 7th Street

Long Beach, CA 90822

Email: Joline.chen@va.gov

Running Title: Pain and Kidney Disease 
Background: Chronic pain is a common condition among the general population. However, large epidemiological studies examining the role of pain in the deterioration of kidney function, development of chronic kidney disease (CKD), and risk of death are lacking.

Study Design: Retrospective cohort study

Setting \& Participants: A nationally representative cohort of 2,360,056 US veterans with baseline estimated glomerular filtration rate $(\mathrm{eGFR}) \geq 60 \mathrm{ml} / \mathrm{min} / 1.73 \mathrm{~m}^{2}$, followed up October 2004-September 2006.

Predictor: 4 pain categories were compared: none (score, 0), mild (1-4), moderate (5-6), or severe $(\geq 7)$.

Outcomes: eGFR decline (determined by eGFR slope) and combined incident eGFR $<60$ $\mathrm{ml} / \mathrm{min} / 1.73 \mathrm{~m}^{2}$ or all-cause death.

Measurements: We examined the pain management pattern and the association of reported pain with (A) rapid eGFR decline, and (B) a composite outcome of incident eGFR $<60 \mathrm{ml} / \mathrm{min} / 1.73 \mathrm{~m}^{2}$ or all-cause death using logistic regression and Cox models adjusted for baseline eGFR, demographics, comorbidities, cardiovascular risk factors, and depression.

Results: Approximately $60 \%$ of veterans reported pain of any severity during the baseline period. The most commonly prescribed analgesics were opioids. In a doseresponse relationship, veterans reporting moderate or severe pain had higher risk of faster eGFR decline compared to those reporting none (ORs of 1.11 [95\% CI, 1.09-1.14] and 1.17 [95\% CI, 1.14-1.21] for moderate and severe pain, respectively). In combined analyses, veterans reporting moderate or severe pain both had 30\% higher incident eGFR 
$<60 \mathrm{ml} / \mathrm{min} / 1.73 \mathrm{~m}^{2}$ or death compared to those reporting none (HRs of 1.30 [95\% CI, 1.28-1.31] and 1.30 [95\% CI, 1.28-1.32] for moderate and severe pain, respectively). Limitations: Lack of granular data regarding the type and location of the pain. Conclusions: We observed a high prevalence of pain and analgesic prescription in the US veteran population with normal eGFR. Pain was associated with higher incidence of eGFR $<60 \mathrm{ml} / \mathrm{min} / 1.73 \mathrm{~m}^{2}$, faster kidney function decline, and higher mortality.

Keywords: chronic Pain, Glomerular Filtration Rate (GFR), Chronic Kidney Disease (CKD), Mortality, renal function, kidney disease trajectory, CKD progression, veterans, analgesics, opioid, nonsteroidal anti-inflammatory drug (NSAID), pain medication 
Chronic non-malignant pain is a common and burdensome condition affecting nearly 100 million Americans. It accounts for $20 \%$ of outpatient visits, $12 \%$ of all prescriptions, and over 100 billion dollars per year in direct costs (such as health care services) and indirect expenses (i.e. lost productivity). ${ }^{1,2}$ In a survey of 28,902 working adults, pain was the most common cause of long-term disability in the United States with over 50 million lost work days per year. ${ }^{3}$ A survey of 6,775 individuals estimated that as much as $19 \%$ of the US adult population suffers from constant or frequent pain greater than 3 months a year. ${ }^{4}$

Despite being a widespread condition, the epidemiology of chronic pain is poorly understood. Chronic pain is reportedly more common in people with comorbidities such as cardiovascular disease, diabetes mellitus (DM), and hypertension. ${ }^{4}$ While chronic kidney disease (CKD) is commonly a consequence of the aforementioned diseases, ${ }^{5}$ chronic pain may also be associated with declining kidney function, progression to CKD, and all-cause death, as it can signal or cause kidney derangement through several possible mechanisms. For example, diabetic and hypertensive patients are at higher risk for neuropathic pain and peripheral artery disease leading to increased non-malignant chronic pain. ${ }^{6-9}$ Furthermore, pain medications such as non-steroidal anti-inflammatory drugs (NSAIDs) are directly associated with kidney injury and may be another risk factor for worsening kidney disease and poor outcomes. ${ }^{10-12}$

There is limited evidence as to whether pain is associated with the risk of developing CKD and subsequent mortality in patients with normal kidney function. Hence, we examined our hypothesis that pain is associated with development of kidney disease, eGFR decline, and long-term mortality in a large, nationally representative contemporary 
cohort of US veterans.

\section{METHODS}

\section{Study Population and Data Source}

Data were obtained from the Racial and Cardiovascular Risk Anomalies in CKD (RCAV) study, which examines risk factors in patients with incident eGFR $<60$ $\mathrm{ml} / \mathrm{min} / 1.73 \mathrm{~m}^{2}$ in US veterans. The RCAV database has been described previously. ${ }^{13} \mathrm{In}$ short, the RCAV cohort is identified using the national Veterans Affairs (VA) Decision Support System National Data Extracts Laboratory Results file to extract data about serum creatinine and identify veterans with normal kidney function on the basis of estimated glomerular filtration rate $(\mathrm{eGFR}) \geq 60 \mathrm{~mL} / \mathrm{min} / 1.73 \mathrm{~m} 2$. The eGFRs in the RCAV study were calculated according to the CKD-EPI (CKD Epidemiology Collaboration) equation. ${ }^{14}$ The present study population is made up of all US veterans with a stable eGFR of $\geq 60 \mathrm{ml} / \mathrm{min} / 1.73 \mathrm{~m}^{2}$ and a documented pain score during the baseline period, from October 1, 2004, through September 30, 2006, and at least 3 creatinine measurements during the follow up period. After excluding 841,423 patients with no baseline pain measurement and 380,999 patients with fewer than three creatinine measurements, the final cohort included 2,360,056 participants. Patients were followed up until they reached a pre-specified end point or were censored at the date of last healthcare or administrative visit, or on July 26, 2013. In combined outcome analyses, patients were followed up until event (first occurrence of eGFR $<60 \mathrm{ml} / \mathrm{min} / 1.73 \mathrm{~m} 2$ or death) or censoring (end of follow-up period), whichever occurred first. Because of the large sample size, the anonymity of the patients studied, and the nonintrusive nature of the research, the requirement for informed consent was waived. The study was approved 
by the institutional review committees at the Memphis (IRB 396977-11) and Long Beach

Veterans Affairs Medical Centers (MIRB 1241).

\section{Clinical and Demographic Measures}

In the VA health system, pain scores were obtained during every veteran's visit along with vital signs according to the VA's "Pain as the Fifth Vital Sign" campaign which started in 1999 and is publicly available and downloadable. ${ }^{15}$ This initiative mandated measurement and documentation of patients' self-reported pain during any clinical encounter. The severity of pain is reported using a Numerical Rating Scale, which is a validated 10-interger verbal and/or visual analog scoring system, where 0 represents no pain and 10 the worst pain experienced. A missing or invalid score was considered as not assessed. When multiple scores were recorded during the 2 year of baseline period, we calculated the mean pain score. The median number of pain measurements used to calculate baseline mean pain score was 5 (interquartile range [IQR], 3-10). The mean pain scores were subsequently categorized into none (score, 0$)$, mild (1-4), moderate (5$6)$, or severe pain $(\geq 7)^{15,16}$.

Socio-demographic characteristics, comorbid conditions, medication data, and laboratory characteristics were obtained, as previously described. ${ }^{17-20}$ Information about age, gender and race were obtained through the VA Corporate Data Warehouse and from Medicare through the VA-Medicare data merge project. $^{21}$ Medication data were obtained from computerized dispensation records from any VA pharmacy. Information about comorbidities was collected from the VA Inpatient and Outpatient Medical SAS Datasets using ICD-9-CM diagnostic and procedure codes and Current Procedural Terminology (CPT) codes. $^{22}$ Prevalent comorbidities were defined as those diagnosed during October 
1, 2004 through September 30, 2006.

\section{Outcomes}

We defined two outcomes: A) eGFR decline (determined by eGFR slope), and B) combined incident eGFR $<60 \mathrm{ml} / \mathrm{min} / 1.73 \mathrm{~m}^{2}$ or all-cause death. Data on all-cause death was obtained from the VA Vital Status Files, which contain dates of death or last medical/administrative encounter from all sources in the VA system, with sensitivity and specificity of $98.3 \%$ and $99.8 \%$, respectively, as compared to the National Death Index. ${ }^{23}$ Decline in eGFR (on incident eGFR $<60 \mathrm{ml} / \mathrm{min} / 1.73 \mathrm{~m}^{2}$ ) was defined as two consecutive eGFRs $<60 \mathrm{ml} / \mathrm{min} / 1.73 \mathrm{~m}^{2}$ separated by $\geq 90$ days, and a $\geq 25 \%$ decrease from the baseline eGFR. ${ }^{24}$ eGFR was calculated from serum creatinine measurements using the CKD-EPI creatinine equation. ${ }^{25}$ Slopes of eGFR were calculated in each patient from all available eGFRs derived from outpatient serum creatinine measurements and using leastsquares regression. The median number of serum creatinine measurements used to calculate eGFR slopes was 11 (IQR, 7-18). Rapid decline was defined as slopes of steeper than $5 \mathrm{ml} / \mathrm{min} / 1.73 \mathrm{~m}^{2}$ per year (i.e., eGFR slope of less than $-5 \mathrm{ml} / \mathrm{min} / 1.73 \mathrm{~m}^{2}$ per year). ${ }^{24}$

\section{Statistical Analysis}

Data were summarized using proportions, means \pm standard deviation, or median (IQR) as appropriate. We used logistic regression models to examine associations between pain categories and odds of rapid eGFR decline. Associations between pain categories and all-cause mortality were assessed using adjusted survival functions based on Cox models, and Cox proportional hazards models for time-to-event analysis. We evaluated the association between pain and CKD progression within the aforementioned 
pain categories, using "none" as reference.

All associations were examined in models adjusted for the following possible confounders: age, gender, marital status, race/ethnicity, income, average body mass index (BMI) and systolic and diastolic blood pressures during the baseline period, baseline eGFR, angiotensin converting enzyme inhibitor use during the baseline period, and the following baseline comorbidities: DM, hypertension, cardiovascular disease (defined as acute myocardial infarction, angina, coronary artery disease, previous coronary artery bypass grafting, percutaneous coronary intervention, congestive heart failure or peripheral vascular disease), cerebrovascular disease, chronic lung disease, rheumatologic disease, hemiplegia, depression, AIDS, dementia, malignancy, liver disease, peptic ulcer disease, and Charlson Comorbidity Index.$^{26}$ In sensitivity analysis, we additionally examined associations after adjustment for use of pain medication and for presence of urine albumin-creatinine ratio (UACR, mg/g). Associations of pain with outcomes were also examined across a priori selected subgroups of age, gender, race, comorbidities and use of pain medications during the baseline period. Statistical analyses were performed using STATA MP version 12 (Stata Corp LP, College Station, TX).

\section{RESULTS}

\section{Baseline Characteristics}

The mean age of the cohort at baseline was $60 \pm 13$ (standard deviation) years. There were $79 \%$ and $17 \%$ white and African-American patients, respectively; $25 \%$ were diabetic and $8.3 \%$ had eGFR decline slope steeper than $5 \mathrm{ml} / \mathrm{min} / 1.73 \mathrm{~m}^{2}$ per year. Baseline characteristics of patients categorized by pain severity are shown in Table $\mathbf{1 .}$ Patients with moderate to severe pain were younger and less likely to be married and they 
had lower income and more history of depression. Alternatively, patients reporting no or mild pain were older, more often non-African American, and have more known history of hypertension and cardiovascular disease. Despite reporting no pain, $6 \%$ and $12 \%$ of patients were prescribed opioids and non-opioids, respectively. The frequency of opioid, non-opioid, and NSAID prescription increased as the severity of pain score increased. A comparison of characteristics among the included and excluded patients showed that differences were clinically insignificant (Table S1, available as online supplementary material).

\section{Deterioration of Kidney Function}

The medians of the eGFR slopes were -0.4 (IQR, -1.9 to 0.9 ), -0.3 (IQR, -2.0 to 1.0 ), -0.3 (IQR, -1.8 to 1.1 ), and -0.2 (IQR, -1.8 to 1.2 ) $\mathrm{ml} / \mathrm{min} / 1.73 \mathrm{~m}^{2}$ per year in the none, mild, moderate and severe pain categories, respectively. There were 195,988 veterans who had eGFR decline slopes steeper than $5 \mathrm{ml} / \mathrm{min} / 1.73 \mathrm{~m}^{2}$ per year. Logistic regression models for eGFR decline slope steeper than $5 \mathrm{ml} / \mathrm{min} / 1.73 \mathrm{~m} 2$ per year showed that worsening pain is incrementally associated with higher likelihood of faster kidney function decline (Table 2, Figure 1) after multivariate adjustment. Patients reporting severe pain had significantly higher adjusted risk of rapid eGFR decline compared to those reporting none (odds ratio [OR], 1.17; 95\% CI, 1.14-1.21). This association persisted across various subgroups of patients (Figure S1A). A sensitivity analysis with slope steeper than $-3 \mathrm{ml} / \mathrm{min} / 1.73 \mathrm{~m} 2$ per year showed similar unadjusted and adjusted results (Table S2). Characteristics of patients with more rapid eGFR decline were similar to those in the overall cohort, with younger, lower income patients reporting moderate to severe pain. 


\section{Combined Outcome}

The median follow-up time was 7.77 (IQR, 5.94-8.38) years. There were 268,326 patients with incident eGFR $<60 \mathrm{ml} / \mathrm{min} / 1.73 \mathrm{~m}^{2}$ and 427,321 deaths. In unadjusted analyses, patients with mild pain had a higher hazard ratio (HR) of reaching the composite end point. However, adjusted analyses showed a significant relationship between pain category and the combined incident eGFR $<60 \mathrm{~mL} / \mathrm{min} / 1.73 \mathrm{~m} 2 /$ death outcome in both survival function curves (Figure 2) and Cox regression models (Table S3, Figure S2). Patients reporting severe pain had a 30\% higher risk of reaching the composite end point compared to those reporting none (HR, 1.30; 95\%CI, 1.28-1.32). The HRs of the combined outcome were similar across all subgroups (Figure S1B).

Sensitivity analyses performed on the individual components of the composite end point showed a similar trend between pain severity and mortality in the adjusted relationship as that in the combined outcome (Figure S2). The adjusted HRs between pain severity and development of different stages of CKD were consistently elevated (Tables S4). We also performed sensitivity analyses adding pain medication to the multivariate analyses and the HRs between pain and combined outcomes remain elevated (tables $\boldsymbol{a}$ and $\boldsymbol{b}$ of Item S1). After adding available UACR data to the multivariate analyses, the trend of elevated HRs between pain and outcome remains the same (Table S5).

\section{DISCUSSION}

We observed that pain was reported in $60 \%$ of the studied US veterans and pain medication use was common. Opioids were prescribed to $40 \%-58 \%$ of patients reporting any degree of pain and to $6 \%$ of patients reporting none. These findings are similar to the 
results of previous reports form the VA. ${ }^{27,28}$ Pain was associated with a more rapid eGFR decline and higher incidence of eGFR $<60 \mathrm{ml} / \mathrm{min} / 1.73 \mathrm{~m}^{2}$ or death after controlling for confounding variables such as age, gender, and various comorbid states.

Pain was previously reported to be associated with all-cause mortality in a number of studies. Macfarlane et al $^{29}$ linked local and widespread pain to $21 \%$ and $31 \%$ higher risks of mortality, respectively, in an eight-year cohort study of 6,569 adults in Northwest England, although malignancy was the main etiology of higher mortality in that study. Also, in a 12-year cohort study of 1,609 Swedish adults, not only was widespread pain associated with a 54\% higher all-cause mortality risk, but also the strongest association of widespread pain was with cardiovascular mortality whereas the association with cancer was not significant. ${ }^{30,31}$ To the best of our knowledge no prior study has researched the association of pain with kidney function derangement. A number of potential mechanisms may explain the hypothesized relationships in our study (Figure S3). For example, rheumatological disease (e.g. rheumatoid arthritis) is painful and associated with increased production of inflammatory cytokines. Maladapative responses to chronic inflammation have been linked with increased tubular secretion of transforming growth factor $\beta$ ), apoptosis, extracellular matrix deposition, transdifferentiation of tubular cells into myofibroblasts, development of interstitial fibrosis, and subsequent GFR decline. ${ }^{32}$ Secondly, DM and hypertension, the leading causes of CKD, are major risk factors for coronary artery disease and peripheral vascular disease, which manifest with ischemic pain. ${ }^{6-9}$ It has also been shown that DM causes neuropathic pain through hyperglycemiainduced nerve damage leading to hyperexcitable peripheral and central pathways of pain. ${ }^{33,34}$ Moreover, hypertension is reportedly associated with increased sensitivity to 
pain in such sizable populations with chronic pain as those with low back pain. ${ }^{35}$ Also, a number of "causal" pathways linking pain to kidney derangements may be possible.

Chronic pain reciprocally causes stress, which in turn leads to the release of catecholamines and glucocorticoids inducing an increase in blood pressure,$^{36,37}$ and the resulting hypertensive nephropathy eventually leads to CKD and the morbidity and mortality associated with it. ${ }^{6}$ Lastly, patients with chronic pain frequently use analgesics including NSAIDs, which are associated with analgesic nephropathy through prostaglandin inhibition, reduced medullary blood flow, free radical formation, direct cell injury, and immunological mechanisms eventually leading to interstitial nephritis, renal papillary necrosis, and CKD. ${ }^{10-12}$ In our study as demonstrated in the sensitivity analyses, the effect between pain and development of CKD is strongest in the mild pain group after adjustment for pain medications, suggesting that pain medication use may have a role in development of CKD. Although we hypothesized that hypertension, DM, cardiovascular disease, and cancers are all associated with pain and may contribute to the development of kidney disease, the association of pain with eGFR decline and incident eGFR $<60$ $\mathrm{ml} / \mathrm{min} / 1.73 \mathrm{~m}^{2}$ remained significant even after adjusting for the effect of these confounders. Thus, it is possible that there may be other unaccounted factors linking the mechanism of pain and development of kidney disease.

We also noted that depression was only reported in $4 \%$ of patients not reporting any pain, but was present in $12 \%-17 \%$ of patients reporting of pain. While pain and depression are commonly co-existent in the general population, depression may be a particularly important risk factor contributing to other mental health disorders including post-traumatic stress disorder and opioid abuse and dependence among the US veterans. ${ }^{27}$ 
In a randomized controlled trial by Kroenke $e t a l,{ }^{38}$ treatment with antidepressants has been suggested as an integral approach to a comprehensive pain management approach.

Our study was limited by the lack of access to more granular data regarding the type and location of the pain. We also did not have the specifics on the details of analgesic medications actually filled or consumed by veterans. In addition, our data comprised mostly male US veterans who are not representative of the US general population. However, this study sheds light on the epidemiology of pain and analgesic use among US veterans and potential consequences of chronic pain using the long term and comprehensive national-level data.

In conclusion, we observed that pain was associated with faster kidney function decline in a dose-response relationship; pain was also associated with higher incidence of eGFR $<60 \mathrm{ml} / \mathrm{min} / 1.73 \mathrm{~m}^{2}$ and mortality. Among US veterans, pain and pain medication use are highly prevalent. Further studies will be needed to better evaluate whether comprehensive treatment of pain would lead to better long-term morbidity and mortality outcomes.

\section{ACKNOWLEDGEMENTS}

Opinions expressed in this paper are those of the authors and do not necessarily represent the opinion or official policy of the Department of Veterans Affairs, Department of Air Force, the Department of Defense, or the US Government.

Support: This study was supported by grant R01DK096920 to Drs Kovesdy and Kalantar-Zadeh and is the result of work supported with resources and the use of facilities at the Memphis VA Medical Center and the Long Beach VA Medical Center. Support for VA/CMS data is provided by the US Department of Veterans Affairs, Veterans Health 
Administration, Office of Research and Development, Health Services Research and Development, VA Information Resource Center (project numbers SDR 02-237 and 98004). The funding sources of this study had no role in study design; collection, analysis, and interpretation of data; writing the report; and the decision to submit the report for publication.

Financial Disclosure: Dr Kalantar-Zadeh has received honoraria from Abbott, Abbvie, Alexion, Amgen, American Society of Nephrology, Astra-Zeneca, Aveo, Chugai, DaVita, Fresenius, Genetech, Haymarket Media, Hospira, Kabi, Keryx, National Institutes of Health, National Kidney Foundation, Relypsa, Resverlogix, Sanofi, Shire, Vifor, and ZS-Pharma. Drs Kovesdy, Chen, and Kalantar-Zadeh are employees of the US Department of Veterans affairs. Dr Sosnov is an employee of the Department of Air Force and the Department of Defense. The other authors declare that they have no other relevant financial interests.

Contributions: Study concept and design: JLTC, S-FA, VR, ES, JAS, CPK, KK-Z; data acquisition: CPK, KK-Z; data analyses/interpretation: VR, JLTC, S-FA, ES, CPK, KK-Z, statistical analysis: VR, ES, supervision or mentorship: CPK, KK-Z, JLTC. Each author contributed important intellectual content during manuscript drafting or revision and accepts accountability for the overall work by ensuring that questions pertaining to the accuracy or integrity of any portion of the work are appropriately investigated and resolved. JLTC and KK-Z take responsibility that this study has been reported honestly, accurately, and transparently; that no important aspects of the study have been omitted; and that discrepancies from the study as planned have been explained.

Peer Review: Evaluated by 2 external peer reviewers, a Statistical Editor, a Co- 
Editor, and the Editor-in-Chief.

\section{REFERENCES}

1. Dzau VJ, Pizzo PA. Relieving pain in America: insights from an Institute of Medicine committee. Jama. 2014;312(15):1507-1508.

2. Alford DP, Krebs EE, Chen IA, Nicolaidis C, Bair MJ, Liebschutz J. Update in pain medicine. J Gen Intern Med. 2010;25(11):1222-1226.

3. Stewart WF, Ricci JA, Chee E, Morganstein D, Lipton R. Lost productive time and cost due to common pain conditions in the US workforce. Jama. 2003;290(18):2443-2454.

4. Kennedy J, Roll JM, Schraudner T, Murphy S, McPherson S. Prevalence of persistent pain in the U.S. Adult population: new data from the 2010 national health interview survey. $J$ Pain. 2014;15(10):979-984.

5. Coresh J, Selvin E, Stevens LA, et al. Prevalence of chronic kidney disease in the United States. Jama. 2007;298(17):2038-2047.

6. Levey AS, Coresh J. Chronic kidney disease. Lancet. 2012;379(9811):165-180.

7. Lee WL, Cheung AM, Cape D, Zinman B. Impact of diabetes on coronary artery disease in women and men: a meta-analysis of prospective studies. Diabetes Care. 2000;23(7):962968.

8. Rosendorff C, Black HR, Cannon CP, et al. Treatment of hypertension in the prevention and management of ischemic heart disease: a scientific statement from the American Heart Association Council for High Blood Pressure Research and the Councils on Clinical Cardiology and Epidemiology and Prevention. Circulation. 2007;115(21):2761-2788.

9. Hirsch AT, Criqui MH, Treat-Jacobson D, et al. Peripheral arterial disease detection, awareness, and treatment in primary care. Jama. 2001;286(11):1317-1324.

10. Gault MH, Barrett BJ. Analgesic nephropathy. Am J Kidney Dis. 1998;32(3):351-360.

11. De Broe ME, Elseviers MM. Analgesic nephropathy. N Engl J Med. 1998;338(7):446-452.

12. Vadivel N, Trikudanathan S, Singh AK. Analgesic nephropathy. Kidney Int. 2007;72(4):517-520.

13. Gosmanova EO, Lu JL, Streja E, Cushman WC, Kalantar-Zadeh K, Kovesdy CP. Association of medical treatment nonadherence with all-cause mortality in newly treated hypertensive US veterans. Hypertension. 2014;64(5):951-957.

14. Stevens LA, Claybon MA, Schmid CH, et al. Evaluation of the Chronic Kidney Disease Epidemiology Collaboration equation for estimating the glomerular filtration rate in multiple ethnicities. Kidney Int. 2011;79(5):555-562.

15. Washington DC: National Pain Management Coordinating Committee. Pain as the 5th Vital Sign Toolkit, revised edition.: Veterans Health Administration; 2000.

16. Serlin RC, Mendoza TR, Nakamura Y, Edwards KR, Cleeland CS. When is cancer pain mild, moderate or severe? Grading pain severity by its interference with function. Pain. 1995;61(2):277-284.

17. Molnar MZ, Kalantar-Zadeh K, Lott EH, et al. ACE Inhibitor and Angiotensin Receptor Blocker Use and Mortality in Patients with Chronic Kidney Disease. Journal of the American College of Cardiology. 2014;63(7):650-658.

18. Kovesdy CP, Lott EH, Lu JL, et al. Hyponatremia, hypernatremia, and mortality in patients with chronic kidney disease with and without congestive heart failure. Circulation. 2012;125(5):677-684.

19. Kovesdy CP, Lott EH, Lu JL, et al. Outcomes associated with microalbuminuria: effect modification by chronic kidney disease. Journal of the American College of Cardiology. 
2013;61(15):1626-1633.

20. VIReC Research User Guide; VHA Medical SAS Inpatient Datasets FY2006-2007. . Hines, IL, U.S. Department of Veterans Affairs. VA Information Resource Center. 2007.

21. US Department of Veterans Affairs VA Information Resource Center Data Quality Update: Race. . 2009.

22. VIReC Research User Guide. VHA Medical SAS Inpatient Datasets FY 2006-2007. Hines IUSDoVAVIRC. 2007.

23. Arnold N SM, Maynard C, Hynes DM. VIReC Technical Report 2: VA-NDI Mortality Data Merge Project. In: Center VIR, editor. Hines, IL. 2006.

24. KDIGO 2012 Clinical Practice Guideline for the Evaluation and Management of Chronic Kidney Disease. Kidney International Supplements. 2013(3):136-150.

25. Levey AS, Stevens LA, Schmid $\mathrm{CH}$, et al. A new equation to estimate glomerular filtration rate. Annals of internal medicine. 2009;150(9):604-612.

26. Deyo RA, Cherkin DC, Ciol MA. Adapting a clinical comorbidity index for use with ICD-9CM administrative databases. J Clin Epidemiol. 1992;45(6):613-619.

27. Edlund MJ, Austen MA, Sullivan MD, et al. Patterns of opioid use for chronic noncancer pain in the Veterans Health Administration from 2009 to 2011. Pain. 2014;155(11):23372343.

28. Mosher HJ, Krebs EE, Carrel M, Kaboli PJ, Weg MWV, Lund BC. Trends in prevalent and incident opioid receipt: an observational study in Veterans Health Administration 20042012. J Gen Intern Med. 2015;30(5):597-604.

29. Macfarlane GJ, McBeth J, Silman AJ. Widespread body pain and mortality: prospective population based study. Bmj. 2001;323(7314):662-665.

30. Andersson HI. Increased mortality among individuals with chronic widespread pain relates to lifestyle factors: a prospective population-based study. Disabil Rehabil. 2009;31(24):1980-1987.

31. Andersson HI. The course of non-malignant chronic pain: a 12-year follow-up of a cohort from the general population. Eur J Pain. 2004;8(1):47-53.

32. Silverstein DM. Inflammation in chronic kidney disease: role in the progression of renal and cardiovascular disease. Pediatr Nephrol. 2009;24(8):1445-1452.

33. Veves A, Backonja M, Malik RA. Painful diabetic neuropathy: epidemiology, natural history, early diagnosis, and treatment options. Pain Med. 2008;9(6):660-674.

34. Boulton AJM, Vinik Al, Arezzo JC, et al. Diabetic neuropathies: a statement by the American Diabetes Association. Diabetes Care. 2005;28(4):956-962.

35. Bruehl S, Chung OY, Jirjis JN, Biridepalli S. Prevalence of clinical hypertension in patients with chronic pain compared to nonpain general medical patients. Clin J Pain. 2005;21(2):147-153.

36. Chapman CR, Tuckett RP, Song CW. Pain and stress in a systems perspective: reciprocal neural, endocrine, and immune interactions. J Pain. 2008;9(2):122-145.

37. Blackburn-Munro G, Blackburn-Munro RE. Chronic pain, chronic stress and depression: coincidence or consequence? J Neuroendocrinol. 2001;13(12):1009-1023.

38. Kroenke K, Bair MJ, Damush TM, et al. Optimized antidepressant therapy and pain selfmanagement in primary care patients with depression and musculoskeletal pain: a randomized controlled trial. Jama. 2009;301(20):2099-2110. 
Table 1: Baseline demographic and clinical characteristics of studied US veterans

\begin{tabular}{|c|c|c|c|c|c|}
\hline \multirow{2}{*}{ Variable } & \multirow{2}{*}{ All } & \multicolumn{4}{|c|}{ Pain } \\
\hline & & None & Mild & Moderate & Severe \\
\hline & $(\mathrm{N}=2,360,056)$ & $(n=943,190)$ & $(\mathrm{n}=1,174,129)$ & $(n=162,257)$ & $(\mathrm{n}=80,480)$ \\
\hline Age $(y)$ & $60 \pm 13$ & $64 \pm 13$ & $59 \pm 13$ & $54 \pm 12$ & $53 \pm 12$ \\
\hline Female sex & 6 & 4 & 7 & 9 & 8 \\
\hline Married & 57 & 63 & 53 & 50 & 48 \\
\hline \multicolumn{6}{|l|}{ Race } \\
\hline White & 79 & 83 & 77 & 74 & 68 \\
\hline African-American & 17 & 13 & 19 & 22 & 27 \\
\hline Hispanic & 2 & 2.0 & 2.2 & 1.7 & 1.9 \\
\hline Other & 2 & 1.9 & 2.2 & 2.7 & 3.1 \\
\hline $\begin{array}{l}\text { Mean annual income, } \\
\$\end{array}$ & $\begin{array}{c}23000 \\
{[12000-} \\
36000]\end{array}$ & $\begin{array}{c}28000 \\
{[14000-} \\
50000]\end{array}$ & $\begin{array}{c}21000[11000- \\
32000]\end{array}$ & $\begin{array}{c}18000 \\
{[11000-} \\
30000]\end{array}$ & $\begin{array}{c}17000 \\
{[10000-} \\
30000]\end{array}$ \\
\hline Baseline eGFR & $83.5 \pm 15.4$ & $80.6 \pm 14.2$ & $84.6 \pm 15.6$ & $88.6 \pm 16.0$ & $89.8 \pm 16.0$ \\
\hline Systolic BP & $136 \pm 19.0$ & $136 \pm 18.9$ & $135 \pm 19.1$ & $135 \pm 18.6$ & $135 \pm 18.8$ \\
\hline Diastolic BP & $77.3 \pm 11.7$ & $76.3 \pm 11.5$ & $77.6 \pm 11.9$ & $78.9 \pm 11.8$ & $79.8 \pm 11.9$ \\
\hline $\operatorname{BMI}\left(\mathrm{kg} / \mathrm{m}^{2}\right)$ & $29.3 \pm 5.68$ & $28.8 \pm 5.11$ & $29.6 \pm 5.92$ & $29.9 \pm 6.28$ & $29.8 \pm 6.41$ \\
\hline \multicolumn{6}{|l|}{ Comorbidities } \\
\hline Hypertension & 62 & 64 & 63 & 54 & 48 \\
\hline Diabetes & 25 & 24 & 26 & 22 & 19 \\
\hline CVD* $^{*}$ & 18 & 16 & 20 & 13 & 10 \\
\hline CBVD & 6 & 5 & 7 & 4 & 3 \\
\hline $\begin{array}{l}\text { Chronic Lung } \\
\text { Disease }\end{array}$ & 18 & 14 & 22 & 20 & 16 \\
\hline Depression & 9 & 4 & 12 & 17 & 15 \\
\hline $\begin{array}{l}\text { Liver and GI } \\
\text { Disease }\end{array}$ & 3 & 2 & 3 & 3 & 3 \\
\hline $\begin{array}{l}\text { Rhemutologic } \\
\text { Disease }\end{array}$ & 1 & 1 & 2 & 2 & 2 \\
\hline All malignancies & 10 & 10 & 11 & 6 & 4 \\
\hline $\begin{array}{l}\text { Cancer (non- } \\
\text { metastatic) }\end{array}$ & 9 & 9 & 10 & 6 & 4 \\
\hline $\begin{array}{l}\text { Metastatic- } \\
\text { malignancy }\end{array}$ & 1 & $<1$ & 2 & 1 & $<1$ \\
\hline $\begin{array}{c}\text { Charlson } \\
\text { Comorbidity Index }\end{array}$ & $1[0-1]$ & $0[0-1]$ & $1[0-2]$ & $0[0-1]$ & $0[0-1]$ \\
\hline \multicolumn{6}{|l|}{ Analgesic Medications } \\
\hline Opioid & 28 & 6 & 40 & 58 & 58 \\
\hline Opioid Antagonist & 0.4 & 0.1 & 0.7 & 0.7 & 0.1 \\
\hline Non-Opioid & 28 & 12 & 37 & 42 & 37 \\
\hline NSAID & 8 & 2 & 10 & 16 & 16 \\
\hline
\end{tabular}

Note: Dichotomous/dummy variables are presented as percentage; continuous variables, as mean \pm standard deviation or median [interquartile range]. All $p$-values for comparisons across pain categories were $<0.001$.

Abbreviations: BMI: body mass index; BP: Blood pressure; eGFR: estimated glomerular filtration rate; NSAID: nonsteroidal anti-inflammatory drug; CVD, cardiovascular disease; CBVD, cerebrovascular disease; Gl, gastrointestinal.

${ }^{*}$ Cardiovascular disease is defined as acute myocardial infarction, angina, coronary artery disease, previous coronary artery bypass grafting, percutaneous coronary intervention, congestive heart failure or peripheral vascular disease. 
Table 2. Association of pain severity with eGFR decline slopes steeper than $5 \mathrm{ml} / \mathrm{min} / 1.73 \mathrm{~m}^{2}$ per year.

\begin{tabular}{|c|c|c|c|c|c|c|}
\hline \multirow{2}{*}{ Pain Category } & \multirow[b]{2}{*}{ No. } & \multirow[b]{2}{*}{ eGFR Slope } & \multirow{2}{*}{$\begin{array}{c}\text { Average eGFR at } \\
\text { time of incident } \\
\text { eGFR }<60\end{array}$} & \multirow{2}{*}{ eGFR Slope $<-5$} & \multicolumn{2}{|c|}{ OR (95\% Cl) } \\
\hline & & & & & Unadjusted & Adjusted* \\
\hline None & 943,190 & $-0.4[-1.9$ to 0.9$]$ & 48.2 & $74,020(7.8)$ & 1.00 (reference) & 1.00 (reference) \\
\hline Mild & $1,174,129$ & $-0.3[-2.0$ to 1.0$]$ & 48.1 & $104,209(8.9)$ & $1.14(1.13-1.16)$ & $1.07(1.05-1.08)$ \\
\hline Moderate & 162,257 & $-0.3[-1.8$ to 1.1$]$ & 48.3 & $12,100(7.5)$ & $0.95(0.93-0.97)$ & $1.11(1.09-1.14)$ \\
\hline Severe & 80,480 & $-0.2[-1.8$ to 1.2$]$ & 48.6 & $5,569(7.0)$ & $0.89(0.86-0.91)$ & $1.17(1.13-1.21)$ \\
\hline
\end{tabular}

Note: Unless otherwise indicated, values given as median [interquartile range] or number (percentage of category).

eGFRs expressed in $\mathrm{mL} / \mathrm{min} / 1.73 \mathrm{~m}^{2}$; eGFR slopes, in $\mathrm{mL} / \mathrm{min} / 1.73 \mathrm{~m}^{2}$ per year.

Abbreviations: $\mathrm{Cl}$, confidence interval; eGFR, estimated glomerular filtration rate; OR, odds ratio

* Models were adjusted for the following covariates: age, gender, marital status, race/ethnicity, income, service connectedness, average body mass index and systolic and diastolic blood pressures during the baseline period, baseline eGFR, angiotensin-converting enzyme inhibitor use during the baseline period, and the following baseline comorbidities: diabetes, hypertension, cardiovascular disease (defined as acute myocardial infarction, angina, coronary artery disease, previous coronary artery bypass grafting, percutaneous coronary intervention, congestive heart failure or peripheral vascular disease), cerebrovascular disease, chronic lung disease, rheumatologic disease, hemiplegia, depression, AIDS, dementia, malignancy, liver disease, peptic ulcer disease, and Charlson Comorbidity Index 
Figure 1. Multivariable adjusted odds ratio (95\% CI) of eGFR decline slope steeper than 5 $\mathrm{ml} / \mathrm{min} / 1.73 \mathrm{~m}^{2}$ per year in other categories of pain severity compared to nonein.

Figure 1 Footnote: * Models were adjusted for the following covariates: age, gender, marital status, race/ethnicity, income, service connectedness, average body mass index (BMI) and systolic and diastolic blood pressures during the baseline period, baseline eGFR, angiotensinconverting enzyme inhibitor use during the baseline period, and the following baseline comorbidities: diabetes, hypertension, cardiovascular disease (defined as acute myocardial infarction, angina, coronary artery disease, previous coronary artery bypass grafting, percutaneous coronary intervention, congestive heart failure or peripheral vascular disease), cerebrovascular disease, chronic lung disease, rheumatologic disease, hemiplegia, depression, AIDS, dementia, malignancy, liver disease, peptic ulcer disease, and Charlson Comorbidity Index $\mathrm{aOR}$, adjusted odds ratio

Figure 2. Association between pain categories and composite outcome of incident eGFR <60 $\mathrm{ml} / \mathrm{min} / 1.73 \mathrm{~m}^{2}$ and all-cause mortality using adjusted Cox proportional regression survival curves

Figure 2 Footnote: * Models were adjusted for the following covariates: age, gender, marital status, race/ethnicity, income, service connectedness, average body mass index (BMI) and systolic and diastolic blood pressures during the baseline period, baseline eGFR, angiotensinconverting enzyme inhibitor use during the baseline period, and the following baseline comorbidities: diabetes, hypertension, cardiovascular disease (defined as acute myocardial infarction, angina, coronary artery disease, previous coronary artery bypass grafting, percutaneous coronary intervention, congestive heart failure or peripheral vascular disease), cerebrovascular disease, chronic lung disease, rheumatologic disease, hemiplegia, depression, AIDS, dementia, malignancy, liver disease, peptic ulcer disease, and Charlson Comorbidity Index 


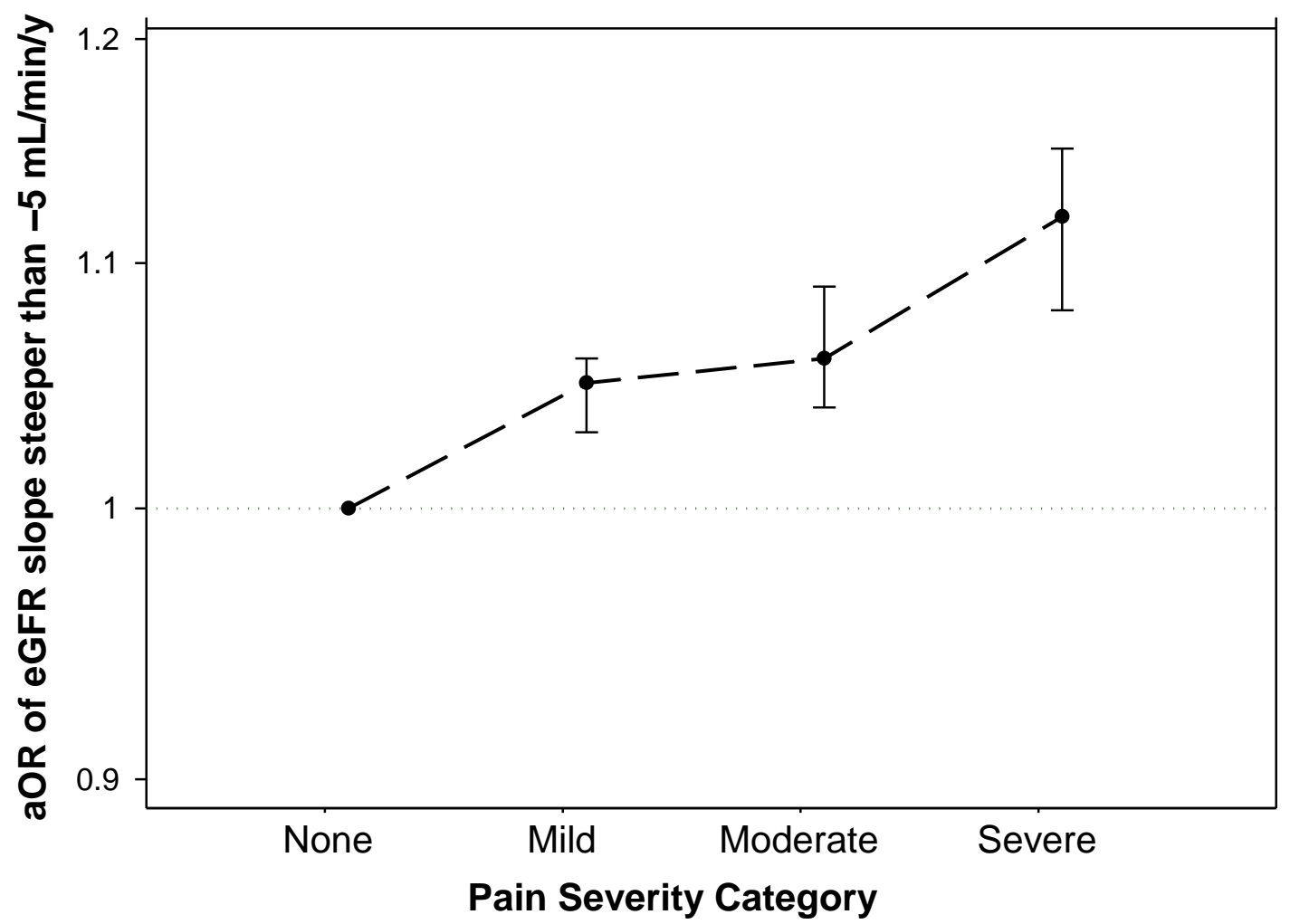




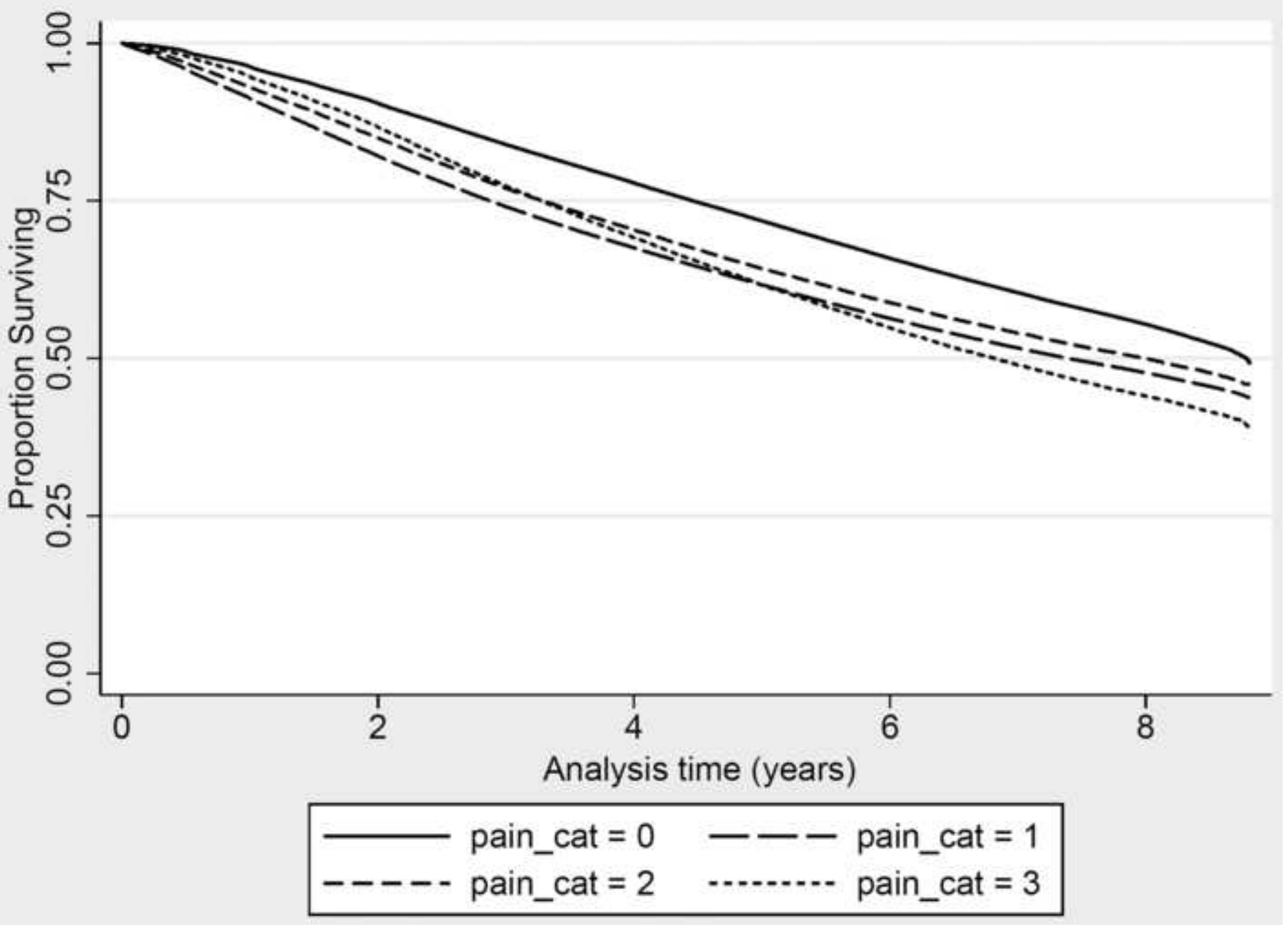

